\title{
A proposal for implementing PBL in programming courses
}

\author{
Sonia Mora Rivera, Mayela Coto Chotto, Georges Alfaro Salazar \\ Escuela de Informática \\ Universidad Nacional \\ Heredia, Costa Rica \\ sonia.mora.rivera@una.cr, mcoto@una.cr, georges.alfaro.salazar@una.cr
}

\begin{abstract}
In the search of teaching strategies more consistent with the current needs of the industry and with the new university pedagogical model, an action research project was carried in the programming courses of the study program of Systems Engineering at the Universidad Nacional of Costa Rica (UNA). The project gradually introduced the principles of autonomy, collaborative learning and shared assessment. This paper reports the experience gained during the three years of the project in terms of a specific proposal for implementing the three principles in the area of programming. Aspects to be considered for an initiative of this kind to be successful are also presented.
\end{abstract}

Keywords- pedagogical approaches, computer education, collaborative learning, autonomy, shared assessment

\section{INTRODUCCION}

Diversas investigaciones en enfoques pedagógicos han demostrado que el aprendizaje basado en problemas y el aprendizaje basado en proyectos contribuyen a resolver parcialmente algunos de los problemas que enfrenta la formación de estudiantes en educación superior [1] [2] [3]. Adicionalmente, estos enfoques reflejan de mejor manera el comportamiento profesional de un ingeniero informático [4].

La Universidad Nacional de Costa Rica (UNA) define en el año 2008 un modelo pedagógico en el cual la enseñanza y el aprendizaje se basan en el análisis y el cuestionamiento de la realidad, en la investigación y el trabajo práctico sobre el contexto en el que un estudiante y su carrera se sitúan, el desarrollo de habilidades para la innovación y la resolución de problemas, la negociación de conflictos, los grupos de trabajo interdisciplinarios, y la toma de decisiones basadas en información confiable y oportuna[5]. A pesar de que el espíritu de este modelo pedagógico es congruente con enfoques pedagógicos como el aprendizaje basado en problemas y el aprendizaje basado en proyectos, su implementación es compleja de llevar a la práctica, principalmente en áreas disciplinarias donde los académicos no poseen formación pedagógica formal.

Es en este contexto, y partiendo de que la capacidad para resolver problemas y trabajar con proyectos son elementos base dentro de la disciplina informática y de que ambos modelos están centrados en el estudiante y se conciben como medios mediante los cual se construye y se comparte el conocimiento y se desarrollan habilidades importantes para la formación integral de los profesionales, que la Escuela de
Informática se propone utilizar un enfoque pedagógico híbrido donde el problema es el estímulo para el aprendizaje y el proyecto el marco en torno al cual este se estructura. Este enfoque es inspirado en el modelo pedagógico POPP (orientación a problemas, organización por proyectos) de la Universidad de Aalborg, Dinamarca [6] y se convierte en el punto de partida para proponer una innovación pedagógica en el plan de estudios de Ingeniería de Sistemas.

En la búsqueda de estrategias didácticas más acordes con el modelo pedagógico de la UNA, se llevó a cabo un proceso de investigación-acción en los cursos de programación de la carrera de Ingeniería en Sistemas de Información, que incorporó de manera paulatina algunos de los principios fundamentales del enfoque POPP. Este artículo presenta, como resultado de dicho proceso, una propuesta de implementación de dichos principios en la carrera de Ingeniería de Sistemas de Información de la Escuela de Informática de la Universidad Nacional. En las siguientes secciones se describirán brevemente los referentes teóricos que le dan el sustento a la propuesta pedagógica presentada, la cual hace énfasis en los tres ejes principales de la misma: autonomía, aprendizaje colaborativo y evaluación. Posteriormente se presenta el diseño de la propuesta desde estos tres ejes y una propuesta de implementación concreta del POPP en los planes de estudio, y finalmente se presentan las conclusiones obtenidas a partir de la experiencia.

\section{FUNDAMENTO TEÓRICO}

El aprendizaje basado en problemas y el aprendizaje orientado por proyectos son dos métodos inductivos de enseñanza ampliamente utilizados [7] [8]. El primero tiene lugar cuando los estudiantes son enfrentados a un problema auténtico como punto de partida al aprendizaje y en el segundo el proceso de aprendizaje se estructura alrededor de un proyecto. En la tabla I se pueden observar las características que tienen en común ambos enfoques. A pesar de que ambos comparten una serie de aspectos comunes, algunos autores [9] identifican diferencias entre los dos enfoques, indicando que usualmente los proyectos requieren de un período mayor de tiempo, y que la gestión de los recursos del proyecto por parte de los estudiantes, así como el manejo de roles, requiere del desarrollo de habilidades muy importantes. Además el trabajo en proyectos es más 
cercano a la realidad profesional de los ingenieros informáticos.

TABLE I. CARACTERÍSTICAS ENFOQUES INDUCTIVOS

\begin{tabular}{|l|}
\hline \multicolumn{1}{|c|}{ Características en común } \\
\hline Centrados en el estudiante \\
\hline Requieren actitud y participación activa del estudiante \\
\hline Multidisciplinarios \\
\hline Basados en la colaboración \\
\hline Logran mayor motivación \\
\hline Mayor responsabilidad en el proceso de aprendizaje \\
\hline
\end{tabular}

En general, el enfoque PBL ha sido analizado en diferentes estudios en el contexto universitario. Las investigaciones lo identifican como un aliado para resolver parcialmente algunos de los problemas que enfrenta la formación de estudiantes en ingeniería informática.. En este enfoque el estudiante se ve motivado a comprender e investigar acerca de un problema para así llegar a proponer una solución adecuada. Prieto [2] señala que este enfoque puede mejorar la calidad del aprendizaje en las universidades pues fomenta competencias como la resolución de problemas, la toma de decisiones, el trabajo en equipo y las habilidades comunicativas, favoreciendo el desarrollo de la capacidad de búsqueda, el manejo de información y de investigación. Kolmos [10] menciona que este enfoque promueve condiciones para el aprendizaje a partir de los procesos de discusión, redacción y la elaboración grupal e individual, facilitando el desarrollo de las competencias de dirección de proyectos y de colaboración, indispensables para los ingenieros informáticos.

Dochy, Segres, Van den Bossche y Gijbels [1] revisaron 43 estudios empíricos de los efectos del PBL en la adquisición de conocimiento y en el desarrollo de habilidades para resolver problemas. Con respecto al primer elemento, los resultados mostraron que el estudiantado adquiere más conocimiento en el corto tiempo, si se utiliza un enfoque de enseñanza tradicional; sin embargo, los estudiantes que experimentaron el PBL fueron capaces de retener el conocimiento adquirido por un período mayor. Con respecto a la adquisición de habilidades, el efecto del enfoque PBL fue positivo en forma contundente.

Nuutila, Törmä y Malmi [11] y Kinnunen y Malmi [12] en estudios realizados sobre la aplicación de PBL en cursos iniciales de programación, usando tanto enfoques PBL como el tradicional, observaron un descenso significativo en la tasa de abandono. Nuutila, Törmä y Malmi [11] indican que, además de programación, los estudiantes adquirieron habilidades relacionadas con el trabajo colaborativo, el estudio independiente y la comunicación de su propio conocimiento. Kinnunen y Malmi [12] mencionan en sus resultados que a los estudiantes les gustan los aspectos sociales del trabajo en grupo, asumir su propio aprendizaje y se sienten motivados por aprender y establecer sus propias metas de aprendizaje y no únicamente por aprobar el curso, lo cual además promueve en ellos la necesidad de investigar. Sin embargo, los autores también mencionan que las dificultades de la dinámica de los grupos, y la incertidumbre que genera la solución de los problemas son muy difíciles de superar para algunos estudiantes.

Similarmente, Hamalainen [13] reporta los resultados obtenidos en un curso de introducción a los conceptos teóricos de ciencias de la computación: los estudiantes que, estando en un enfoque PBL, abandonan el curso lo hacen en las primeras semanas, una vez que entienden de qué se trata el curso contrariamente, los abandonos en el curso bajo un modelo tradicional se dan usualmente después del primer examen. La investigación concluye que los estudiantes generan un mayor compromiso con un curso PBL que con uno de corte tradicional.

Es claro, que el adoptar un método de enseñanza como el PBL implica dejar de lado la enseñanza tradicional y apegarse a un enfoque en el cual las actividades interdisciplinarias se promueven y estimulan el aprendizaje colaborativo y centrado en el estudiante.

\section{A. Ejes principales de la propuesta de innovación pedagógica}

La innovación pedagógica propuesta busca generar cambios en la forma de abordar los cursos de la carrera. Es sabido que los cambios implican tiempo, tanto académicos como estudiantes deben ajustar su cultura y se requieren procesos de asimilación de las nuevas estrategias didácticas. En consecuencia, se propone el cambio como un proceso fundamentalmente basado en experiencias de aprendizaje positivas tanto para los docentes como los estudiantes [14].

Esta hace énfasis especialmente en tres ejes fundamentales: (1) promover la autonomía de los estudiantes; (2) fomentar el aprendizaje colaborativo; y (3) generar procesos de evaluación participativos y alineados con el modelo pedagógico de la UNA.

\section{1) Autonomía}

Ser una persona autónoma implica saber adaptarse a nuevas situaciones y ambientes, buscar recursos y aprender rápidamente para resolver nuevos problemas o manejar nuevos puestos de trabajo y situaciones que enfrenten. Estas habilidades son fundamentales en una sociedad siempre cambiante, donde la única constante es el cambio [15] En este sentido, proporcionar a los estudiantes la oportunidad de participar en procesos que desarrollen su autonomía parece ser la única manera de prepararlos para enfrentar el futuro y al mismo tiempo contribuir a desarrollar las competencias que actualmente la sociedad requiere.

El currículo de Ciencias de la Computación 2013 [16] establece que los programas de estudios universitarios deben preparar a los estudiantes para el aprendizaje permanente, de tal forma que sean capaces de tener éxito en un campo disciplinario de rápida evolución. Los individuos autónomos son valorados por los empleadores de todo el mundo, quiénes 
indican requerir graduados que están dispuestos a aprender, que tengan motivación para trabajar, que sean eficaces en el trabajo en grupo, y que tengan buenas habilidades de comunicación, capacidad para resolver problemas y la capacidad de aprendizaje permanente [17] [18] [19] . Algunos investigadores [20] [21][22] también han argumentado sobre la necesidad de desarrollar aprendices autónomos que puedan responder exitosamente a una economía global y a la sociedad del conocimiento. Como puede verse la industria necesita graduados universitarios que hayan sido formados como pensadores autónomos y críticos. Acorde con esto, muchos programas universitarios en el área de la informática, introducen algunas actividades curriculares que promueven la autonomía, sin embargo estas suelen estar presentes en los últimos años de los programas de pregrado, lo que no favorece un proceso continuo de madurez, de toma de decisiones y de apropiación del propio proceso de aprendizaje.

De acuerdo con [23] hay cuatro áreas de posible toma de decisiones para principiantes: las reglas a seguir en la clase, el contenido del curso, las actividades del curso y los procesos de evaluación. Es claro que, en la mayoría de las ocasiones, los docentes no pueden dejar que los estudiantes decidan significativamente sobre el contenido de los cursos principales de la carrera, ni tampoco pueden perder el control sobre los principales componentes del proceso de evaluación, no obstante, y con el objetivo de desarrollar la madurez intelectual, la capacidad de aprendizaje y la responsabilidad necesaria para ser aprendices autónomos, los estudiantes deben ser expuestos a ambientes que favorezcan el ejercicio de algún nivel extra de decisión, y es de esperar que estos niveles de decisión se incrementen conforme se avance en el plan de estudios, es decir el nivel de decisión de los estudiantes de un curso de tercer nivel de carrera debiera ser mayor que el de los estudiantes en el curso inicial.

Bajo este escenario, es relevante entonces, plantearse la interrogante de ¿cómo diseñar ambientes de aprendizaje que puedan fomentar la autonomía de los estudiantes desde sus primeras experiencias universitarias? Desafortunadamente, los métodos de enseñanza tradicionales comunes en las universidades, en las que los docentes controlan la mayoría de los procesos mediante los cuales los estudiantes aprenden, no son adecuados para lograr este resultado [24]. En el método deductivo tradicional de enseñanza y como [23] argumenta, los docentes controlan la mayoría, si no todos estos procesos. Ellos deciden el contenido que los estudiantes aprenden en el curso, la velocidad a la que ese contenido es cubierto, las tareas y exámenes a través de las cuales se puede dominar la materia, y las condiciones para evaluar el aprendizaje que se ha producido, $\mathrm{y}$ esto afecta negativamente la motivación, la confianza y el entusiasmo por el aprendizaje y no desarrolla la capacidad de aceptar la responsabilidad y la capacidad de tomar la iniciativa. En esta línea, la propuesta pedagógica plantea la autonomía como un eje central de la innovación pedagógica, dado que concordamos con [25] cuando argumenta que la responsabilidad de todos los docentes es promover la autonomía y el pensamiento crítico que contribuya a formar miembros responsables de la sociedad, siendo el contenido de las lecciones un objetivo secundario en este esfuerzo.

\section{2) Aprendizaje colaborativo}

Los trabajos en grupo forman parte común de la educación en informática, sin embargo esta práctica no implica que haya aprendizaje colaborativo. El aprendizaje colaborativo tiene como meta fomentar en los estudiantes la construcción de conocimiento mediante exploración, negociación, y discusión [26]. Además, [27] indica que el aprendizaje colaborativo genera espacios para la confrontación de múltiples perspectivas y la negociación en un proceso de aprendizaje que conduce al desarrollo integral de los participantes.

En [28] se mencionan como fortalezas del aprendizaje colaborativo: (1) la interdependencia positiva (se persigue un objetivo común, el esfuerzo individual beneficia a todo el grupo); (2) la promoción a la interacción (apoyo y ayuda mutua); (3) la responsabilidad individual (aporte individual al aprendizaje de todos); (4) las habilidades interpersonales y grupales (comunicación, toma de decisiones y resolución de conflictos); y (5) la interacción positiva (respeto mutuo, críticas constructivas, reflexión). Cuando hay aprendizaje colaborativo cada uno de los miembros del grupo debe estar comprometido con la meta del grupo, y ser consciente de que su contribución al grupo no es competitiva sino que forma parte de una interdependencia positiva donde el logro de la meta conjunta es más importante que las contribuciones individuales [29].

En este sentido, la propuesta se basa fuertemente en promover dichas actitudes y habilidades en los estudiantes, particularmente mediante el trabajo grupal en la resolución de proyectos acompañados de estrategias de autoevaluación y evaluación por pares.

\section{3) Evaluación}

En el contexto de un enfoque pedagógico como el POPP se plantean dos retos para los procesos de evaluación. Primero, se debe dar un sentido diferente a la conceptualización que los docentes tienen sobre este proceso, y segundo, se debe descentralizar el proceso evaluativo, a fin de promover una mayor participación de los estudiantes [30]. En este sentido, los docentes deben entender que la evaluación tiene una intencionalidad formativa donde es tan importante el proceso como el producto. Paralelamente deben ceder espacio a los estudiantes para que participen en los procesos de evaluación y de esta forma logren una mejor comprensión de su rol como estudiantes responsables y comprometidos con su proceso de aprendizaje y su formación profesional. Involucrar a los estudiantes en estos procesos es necesario para mantener la coherencia curricular entre los objetivos educativos, las actividades de aprendizaje y el sistema de evaluación que 
promueve el POPP. En [31] se refiere a esta coherencia con el concepto de "aprendizaje alineado".

La participación de los estudiantes en los procesos de evaluación, se da usualmente a través de estrategias de autoevaluación y evaluación entre pares. La autoevaluación contribuye al desarrollo de la capacidad crítica, favoreciendo la independencia y creatividad, y la evaluación de pares propicia la elaboración de compromisos, dado que cada estudiante se compromete con los restantes y con el proceso mismo de aprendizaje [30]. Además el uso de estas estrategias promueve la autonomía de los estudiantes y contribuye a la formación de personas responsables.

Desafortunadamente, muchos de los docentes se niegan a adoptar estas estrategias de evaluación aduciendo argumentos como que los estudiantes no están preparados y son irresponsables. Sin embargo, [32] menciona una serie de aspectos en que los estudiantes pueden emitir juicios de valor, tales como: comprensión de los aprendizajes logrados, participación activa o pasiva en la construcción de sus aprendizajes, interés por aprender, aportes al trabajo colaborativo, respeto por las opiniones de las demás, desempeño de los compañeros y calidad de los trabajos. En concreto, la evaluación es una parte muy importante del proceso de enseñanza aprendizaje, es un recurso que permite mejorar la calidad de la enseñanza y de los aprendizajes [33] y por ende es un proceso en el que los estudiantes deben tener participación.

\section{DISEÑO DE LA PROPUESTA}

En esta sección se definen los alcances de la propuesta desde cada uno de los ejes fundamentales mencionados anteriormente. Además se contextualiza el proceso de intervención educativa y se brindarán sugerencias concretas de cómo implementarlos en los distintos cursos.

\section{A. Contextualización}

El proyecto se llevó a cabo durante tres años. En el primer año se realizaron múltiples actividades de formación e intercambio docente conducentes a comprender y utilizar los principios del POPP, y a fortalecer en los académicos las competencias necesarias para diseñar ambientes de aprendizaje que pudieran promover en los estudiantes el desarrollo de las competencias requeridas por la sociedad actual. En los años siguientes el proyecto se enmarca en un proceso de investigación-acción que permite mediante un ciclo de investigación, acción y reflexión evaluar de forma colaborativa continuamente los principios de diseño y ajustar los mismos al resultado de su implementación en el contexto y a los objetivos perseguidos por la intervención [34]. En este sentido, en cada ciclo lectivo se van considerando los cambios necesarios para ir incorporando los principios del POPP en los cursos de programación del plan de estudios, retomando las acciones positivas de cada ciclo lectivo, para ser consideradas en el siguiente.

\section{B. Características de la intervención}

Se realizaron cinco intervenciones a lo largo de los años que duró el proyecto. La Tabla II presenta un resumen de las mismas y los ciclos en las cuales estas fueron llevadas a cabo, además los cursos que fueron intervenidos y la cantidad de personas involucradas, tanto estudiantes como profesores.

TABLE II. INTERVENCIONES REALIZADAS II CICLO 2011 - II CICLO 2013

\begin{tabular}{|c|l|l|c|}
\hline $\begin{array}{c}\mathbf{N}^{\circ} \\
\text { Int. }\end{array}$ & \multicolumn{1}{|c|}{ Cursos afectados } & \multicolumn{1}{c|}{$\begin{array}{c}\text { Población } \\
\text { participante }\end{array}$} & Fecha \\
\hline $1^{\circ}$ & EIF201 Programación I & $\begin{array}{l}150 \text { estudiantes y } 5 \\
\text { docentes }\end{array}$ & II ciclo 2011 \\
\hline $2^{\circ}$ & $\begin{array}{l}\text { EIF200 Ftos Inf. } \\
\text { EIF204 Programación II }\end{array}$ & $\begin{array}{l}350 \text { estudiantes y 8 } \\
\text { docentes }\end{array}$ & I ciclo 2012 \\
\hline $3^{\circ}$ & $\begin{array}{l}\text { EIF 201Programación I } \\
\text { EIF206 Programación III }\end{array}$ & $\begin{array}{l}300 \text { estudiantes y 7 } \\
\text { docentes }\end{array}$ & II ciclo 2012 \\
\hline $4^{\circ}$ & $\begin{array}{l}\text { EIF200 Ftos Inf. } \\
\text { Evaluación Prog. I y } \\
\text { Prog. II }\end{array}$ & $\begin{array}{l}300 \text { estudiantes y 12 } \\
\text { docentes }\end{array}$ & I ciclo 2013 \\
\hline $5^{\circ}$ & EIF200 Ftos Informática. & 60 estudiantes & II ciclo 2013 \\
\hline
\end{tabular}

La primera intervención tiene lugar durante el segundo ciclo lectivo del 2011 en el curso EIF201 Programación I. Esta intervención se realizó al mismo tiempo que se brindaba la capacitación a los académicos, por lo que para los efectos del proyecto se consideró una intervención exploratoria que permitió brindar algunos indicadores útiles para plantear las intervenciones subsiguientes.

En cada intervención se trabajó con los docentes desde antes de iniciar el ciclo lectivo, proponiendo distintas modalidades a considerar en la forma de abordar los contenidos y en los proyectos. Durante el proceso recibían un acompañamiento en el que se brindaban ideas o sugerencias de cómo abordar cada una de las dificultades que surgían en el camino. Cada una de las intervenciones fue evaluada al final del ciclo lectivo mediante cuestionarios, grupos focales y entrevistas a los estudiantes. Adicionalmente, se realizaron grupos focales con los docentes que permitieron compartir las experiencias y enriquecer las subsiguientes intervenciones. Más detalles de la implementación del proyecto y los resultados obtenidos en las intervenciones realizadas pueden encontrarse en [35] [36] [37] [38].

\section{Características de la implementación del POPP}

Como se ha mencionado con anterioridad, en un enfoque pedagógico orientado a problemas, el "problema a resolver" es el estímulo para el aprendizaje, y si partimos de que el problema debe tener relación con la disciplina y poner en contacto al estudiante con su futuro laboral y profesional, este debe también promover el razonamiento complejo y crítico de los estudiantes, la necesidad de investigar y buscar información para encontrar una solución viable, y fortalecer las competencias del trabajo grupal y autónomo. En este 
sentido, el problema se considera el eje central del diseño de la propuesta, ya que a partir de su formulación pueden generarse los restantes componentes de la intervención: aprendizaje colaborativo, autonomía y evaluación participativa.

De acuerdo con lo anterior, el diseño de las intervenciones realizadas en cada uno de los cursos de programación contempló las siguientes características:

\section{1) "Problemas" como estímulo para el aprendizaje}

En el caso de la disciplina informática, generalmente la solución de problemas se concretiza mediante la formulación, la ejecución y la presentación de un proyecto programado, donde el producto final es, en la mayoría de los casos, un programa computacional que resuelve, de alguna manera, la problemática identificada. A partir de "un buen problema" se pueden articular todos los otros principios del enfoque POPP, y el proyecto se convierte en el medio mediante el cual los estudiantes buscan soluciones viables para resolverlos.

Al ser el problema un vehículo a través del cual los estudiantes obtienen conocimiento y adquieren las habilidades deseadas en el curso [2], este debe tener una relación estrecha con los objetivos de aprendizaje. Es deseable que un problema incluya contenidos de otros cursos previos o que el estudiante está llevando conjuntamente, así el problema contribuye a explorar vínculos entre las distintas áreas de la ingeniería informática. Además el problema no debe ser fácil de resolver, no todos sus elementos deben ser conocidos, debe tener varias soluciones y, en la medida de lo posible, integrar enfoques de más de una disciplina. Según [39], estos problemas fomentan la construcción activa del conocimiento y poseen el sabor de la realidad, ya que usualmente no presentan una única solución. Es importante que no se evalúe la solución de los estudiantes por el hecho de que coincida con la del docente, sino por su calidad, creatividad y viabilidad. Esto conlleva a promover un cambio en la cultura, tanto de docentes como de estudiantes, porque un problema poco estructurado puede ser percibido por los estudiantes como falta de conocimiento o de esfuerzo por parte de los docentes, y esto podría repercutir negativamente en la evaluación del desempeño docente.

Se recomienda entonces que los proyectos de los cursos formulen problemas acerca de situaciones reales que abarquen los temas de los cursos y que ejerciten lo que los estudiantes tendrán que hacer en su futuro profesional, de esta manera integran la teoría con la práctica. El campo específico de la ingeniería informática es muy rico en este sentido, ya que el perfil laboral de sus egresados es muy amplio, sin embargo esta no es una tarea fácil, y el planteamiento y búsqueda de "buenos problemas" se vuelve una actividad que requiere de mucho tiempo y trabajo de los docentes. En este contexto, el diseño de la intervención educativa se centra en ir generando, en estudiantes y docentes, una nueva cultura hacia la resolución de problemas. Claramente esta labor inicia con los docentes, ya que ellos son los responsables de la mediación en el aula y tienen un rol fundamental en este proceso.

\section{2) Aprendizaje colaborativo}

Una característica importante del enfoque POPP y de especial interés en esta propuesta es el trabajo colaborativo grupal. En los proyectos es muy común el "divide y vencerás", donde los integrantes del grupo se "dividen" el trabajo del proyecto, en secciones funcionales, y casi nunca media en la división algún objetivo didáctico. En este sentido se propone un trabajo intensivo con los estudiantes fomentando la realización de proyectos en grupo, que fomenten más el trabajo colaborativo.

Además en los cursos predomina la realización de proyectos en parejas o cuando mucho en grupos pequeños con un máximo de 3 participantes por grupo. Tanto la mayoría de los docentes como los estudiantes se sienten cómodos con este tamaño de grupo debido a que sienten que es más sencillo ejercer control sobre el trabajo y esfuerzo realizado por cada miembro del grupo. Sin embargo, las intervenciones realizadas en varios de los cursos de programación como parte del proyecto pusieron en evidencia algunos problemas relacionados a este tamaño de grupo: (1) la falta de un proceso de realimentación oportuna y continua por parte del docente. Ante esto, los docentes argumentaron falta de tiempo para darle seguimiento a 13-15 grupos de estudiantes (en un aula entre 25 y 30 estudiantes); y (2) poco desarrollo de las capacidades de negociación y manejo de conflictos por parte de los estudiantes. Una posible solución a los dos aspectos mencionados, y que además está alineado al espíritu del POPP, es trabajar con grupos de 4 a 5 estudiantes. Esto implicaría que en una clase se tendrían de 5 a 6 grupos en lugar de los 13-15 usuales, por los que el docente puede brindar a los grupos una atención más oportuna y continua durante el desarrollo del proyecto. Adicionalmente, el trabajo en grupos más grandes demanda que los estudiantes desarrollen en mayor medida sus capacidades de organización, negociación, toma de decisiones, distribución de responsabilidades y manejo de conflictos.

De esta manera al diseñar problemas que promuevan el verdadero trabajo colaborativo, se promueve que cada uno de los estudiantes se haga responsable de su desempeño individual dentro del equipo, pero a la vez se estimula la interdependencia positiva, en el sentido de que los estudiantes deban depender los unos de los otros para lograr la meta común. Esta forma de trabajo debe ir acompañada de un proceso de formación que les permita a los estudiantes de informática desarrollar las habilidades necesarias para que el grupo funcione en forma efectiva, tales como el liderazgo, la reflexión crítica y la solución de conflictos. 


\section{3) Autonomía}

En nuestra perspectiva, fomentar la autonomía del estudiante en la carrera implica proporcionarles un ambiente de aprendizaje que les ofrezca la oportunidad de tomar al menos un cierto control de su aprendizaje. Es evidente que desarrollar aprendices autónomos representa un reto tanto para estudiantes como para docentes. Los estudiantes llegan a la universidad provenientes de diversos entornos sociales, culturales y educativos, que influyen en su actitud hacia el proceso de aprendizaje y afecta el grado en que son capaces de convertirse en estudiantes autónomos [40] [41].

Como primer aporte, la intervención diseñada en este proyecto se propuso brindar a los estudiantes niveles de decisión en las actividades del curso (como por ejemplo el tipo de proyecto a realizar, brindar diferentes opciones de proyecto a seleccionar, selección de la manera de presentarlo, etc) y asumir una mayor parte en los procesos de evaluación, particularmente en torno a los proyectos de los cursos. También propuso darles la oportunidad de aprender gradualmente a usar los procesos de auto-evaluación $y$ evaluación por pares como una herramienta eficaz para evaluar el aprendizaje y la participación en el trabajo de grupo.

\section{4) Evaluación participativa}

Como se mencionó anteriormente, con el fin de fomentar la colaboración, negociación y liderazgo, se propone experimentar con grupos de más estudiantes. Para acompañar este proceso, es necesario incorporar estrategias de autoevaluación y evaluación por pares, que les permita a los estudiantes reflexionar críticamente sobre su proceso de aprendizaje y sobre su aporte y el de sus compañeros de grupo a las metas conjuntas.

Estas estrategias contribuyen a cambiar la idea prevaleciente de que la evaluación es exclusivamente una actividad docente. La responsabilidad compartida entre profesores y estudiantes en el proceso de aprendizaje debe promoverse desde los primeros cursos para que paulatinamente los estudiantes adquieran las habilidades necesarias para ir tomando más responsabilidad sobre su proceso de aprendizaje. Las mismas se convierten en instrumentos que desarrollan la habilidad de los estudiantes de evaluar su propio progreso y hacer los ajustes apropiados en forma oportuna. Además, cuando los estudiantes hacen la evaluación por pares aprenden a analizar críticamente su trabajo y el de los otros, incrementando así su compromiso con el trabajo en grupo.

En este sentido el proyecto promovió fuertemente estos procesos en los cursos iniciales, con la expectativa de ir formando a los estudiantes en la adquisición de responsabilidades y evaluación crítica y reflexiva. Idealmente estos procesos deben continuarse en los siguientes cursos, para que los estudiantes adquieran la madurez necesaria en el ámbito de la evaluación.

\section{PROPUESTA CONCRETA}

Esta sección tiene como objetivo proponer la aplicación de los principios del POPP de manera concreta como estrategia pedagógica en los cursos del área de programación del plan de estudios de la carrera de Ingeniería en Sistemas de Información de la Universidad Nacional, sin excluir que los principios y estrategias aquí propuestas puedan ser analizados para otros cursos, áreas y disciplinas. La propuesta se basa en la experiencia obtenida a través de la intervención realizada como parte del proyecto durante los períodos: II ciclo lectivo 2011, I y II ciclo del año 2012 y I ciclo lectivo 2013 (ver Tabla II). La misma es formulada de acuerdo a las posibles acciones que se pueden llevar a cabo y de la cantidad de esfuerzo que pueda involucrarse en esta iniciativa. Particularmente, se presenta una propuesta a corto plazo que le da seguimiento al proyecto de investigación. Una propuesta a mediano plazo puede ser inferida de [38]

Las acciones a corto plazo van orientadas a proponer distintos esquemas de incorporación de los principios del POPP, según el nivel del curso. Sin embargo en este punto es importante aclarar que para lograr un impacto más positivo $\mathrm{y}$ permanente en el plan de estudios es fundamental que se generen directrices claras de las autoridades de la unidad académica hacia docentes y estudiantes que garanticen una adecuada integración de dichos principios en todos los cursos y por todos los docentes de cada cátedra de programación.

\section{A. Cursos iniciales}

Se consideran como cursos iniciales, aquellos en los que los estudiantes tienen sus primeros contactos con la programación. Es decir aquellos en los que se aprende la lógica detrás de la resolución de problemas computacionales y se tiene un primer acercamiento a los lenguajes de programación. En este sentido, se sugiere que el objetivo en los cursos iniciales de la carrera se centre en el aprendizaje colaborativo, como tal el trabajo en grupo en pequeños retos y proyectos se concibe como una estrategia para que los estudiantes desarrollen interdependencias positivas, acompañado de un proceso de evaluación que les haga asumir su responsabilidad y compromiso. Por lo tanto:

- Se debe orientar a los estudiantes sobre el aprendizaje colaborativo, ¿qué significa?, ¿qué compromisos se adquieren?, ¿cómo ponerlo en práctica? etc. Esto es fundamental para que tengan claro que se espera de ellos. De igual manera se les deben brindar estrategias para el manejo de conflictos, como ejemplo: se les indica que un grupo nunca se disuelve, salvo casos excepcionales; se les sugiere que ellos mismos elaboren una "Carta de Compromisos", donde cada grupo acuerda sus obligaciones, sus derechos, sus motivaciones, sus penalizaciones etc. 
- Para el manejo de los temas introductorios de cada curso que por lo general se cubren en una lección o menos, se sugiere que el docente los abarque mediante la asignación de pequeños retos que motiven a los estudiantes, los cuales pueden ser abordados mediante la realización de vídeos, infografías, dramatizaciones, líneas de tiempo, etc. Por ejemplo, para el primer curso de programación se asignaron los siguientes retos con buenos resultados: (1) el tema de deberes y derechos de los estudiantes universitarios puede estudiarse mediante "casos reales" que los estudiantes analizan en grupo con el apoyo de los reglamentos y luego dramatizan al resto de la clase; (2) la historia y generaciones de los computadores es un tema adecuado para que los estudiantes construyan en forma creativa una línea de tiempo; (3) el tema de organización, y componentes del computador puede estudiarse de diversas maneras: mediante la creación de un video; mediante la compra hipotética y justificada de un computador; o mediante la construcción de un documento en Google Drive. Todos los retos anteriores fortalecen el trabajo colaborativo y a la vez le permiten a docentes y estudiantes abarcar de manera diferente los tópicos mencionados. Se sugiere que el docente forme grupos de 4-5 estudiantes y que los vaya rotando para cada uno de los retos, de tal manera que la mayoría de estudiantes tenga la oportunidad de conocerse.

- Para temas de mayor peso que abarcan una parte importante de los contenidos del curso, se propone realizar pequeños proyectos, para ello se sugiere un reto por tema, por ejemplo en el primer curso de programación se sugiere un proyecto para el tema de ciclos y otro para el tema de arreglos. En este caso, se parte del hecho de que los estudiantes ya se conocen por su trabajo con diferentes compañeros en los retos anteriores por lo que se sugiere que sean los estudiantes los que decidan la conformación de los grupos (4-5 miembros). En al menos uno de los proyectos (por ejemplo para el tema de arreglos) se sugiere brindar a los estudiantes tres opciones o variaciones de proyecto para que los estudiantes puedan seleccionar el que más les interese y eso logre motivarlos, así como minimizar las posibilidades de copia entre grupos, y por el contrario fomentar el compartir "su" solución entre ellos y promover los procesos de autonomía. También se puede pensar en proponer el desarrollo de proyectos pequeños, que vayan conformando la solución a un problema mayor, en este caso los docentes deben formular pequeños problemas parciales según los contenidos que el curso va cubriendo, e irlos consolidando en una solución a un problema mayor. Los docentes deben formular de manera concreta y definida cada una de las etapas del problema, de manera que aunque sean pequeñas soluciones estas conllevan a una completa solución al problema global.

\section{B. Cursos de nivel medio}

Los cursos del nivel medio son aquellos en que los estudiantes ya han adquirido los principios básicos de la resolución de problemas en algún lenguaje de programación (usualmente los cursos de programación correspondientes a segundo año de carrera), Desde el enfoque propuesto se propone que en estos cursos se fomente más fuertemente la autonomía de los estudiantes y la toma de decisiones, además de continuar desarrollando la evaluación participativa, y el proceso de aprendizaje colaborativo, de esta manera el trabajo en proyectos como tal debe seguir siendo promovido ya que el mismo, al igual que los cursos anteriores, se concibe como una estrategia para que los estudiantes desarrollen interdependencias positivas, acompañado de un proceso de evaluación que les haga asumir su responsabilidad $\mathrm{y}$ compromiso. Por lo tanto:

- Para el logro de los objetivos del curso se propone realizar un único proyecto, dividido en etapas, cada etapa va aportando de manera parcial a la solución del problema. Se propone presentarle al estudiante de 3 a 5 opciones diferentes de proyecto, ya sean juegos o aplicaciones administrativas, que les permita decidir en qué tipo de proyecto quieren involucrarse y desarrollar a lo largo del curso. Como ejemplo se les propuso a los estudiantes realizar una aplicación para: un restaurante, una ferretería, o una clínica dental, y se les dieron requerimientos mínimos que debía cumplir cada uno. Esto fomenta su autonomía y motivación por el aprendizaje. Los docentes deben formular los proyectos cuidadosamente para asegurar que cada opción cumpla con los objetivos de aprendizaje del curso. Además, deben ser propuestas de problemas abiertos, poco definidos y poco estructurados de manera que el estudiante tenga que ir definiendo y replanteándose todo lo aprendido hasta este momento.

- Bajo este esquema de etapas, la realimentación oportuna del docente es fundamental para la buena ejecución del proyecto y del trabajo colaborativo. Los errores en cada etapa deben ser muy bien canalizados a fin de que los estudiantes logren una adecuada solución final, y sientan motivación por el aprendizaje obtenido y los logros alcanzados parcialmente.

\section{Cursos de niveles superiores}

Desde el enfoque propuesto, se persigue que en los cursos de programación de mayor nivel, más específicamente en cursos de tercero y cuarto año de carrera, se fomente el 
contacto con la industria y se siga fomentando la autonomía, $\mathrm{y}$ aunque se ha venido trabajado la evaluación participativa, $\mathrm{y}$ el proceso de aprendizaje colaborativo, se deben mantener, y de esta manera el trabajo en proyectos como tal debe seguir siendo el centro del aprendizaje. Por lo tanto:

- Se propone realizar, en forma grupal, un sólo proyecto, por etapas, pero a este nivel ya el estudiante está en capacidad de elegir, asociado a su entorno, el problema a desarrollar en el curso, de esta manera él debe buscar, discernir, elegir y desarrollar su propia propuesta de solución a un problema. Esto fomenta su autonomía y motivación por el curso y le permite poner en práctica su conocimiento en el desarrollo de un problema de la vida real.

- $\quad$ El docente debe definir una serie de requisitos que el proyecto debe cumplir para guiarlos en este proceso de selección. Además los docentes deben estar capacitados para orientar adecuadamente al equipo de trabajo en la escogencia del proyecto, para que éste cumpla con los objetivos del curso, es posible que el alcance del proyecto involucre conocimientos adquiridos de otros cursos, disciplinas, etc, y se debe encontrar la manera adecuada de suplir las necesidades de los estudiantes en esta línea.

\section{Generalidades}

Además de las particularidades presentadas anteriormente para los diferentes cursos, de acuerdo a su nivel, el proyecto identificó algunos aspectos de orden general que deben ser considerados a la hora de implementar el enfoque POPP en cada uno de los cursos y que son enunciados a continuación:

- La realimentación oportuna y continua que hace el docente durante el proceso de aprendizaje, es de gran importancia. El docente debe proporcionar, idealmente en el tiempo de aula, el espacio para darle seguimiento al trabajo de los estudiantes en los proyectos, tanto desde el punto de vista de la resolución del problema planteado como de la evolución del trabajo en grupo. Este último aspecto es muy importante ya que usualmente el docente se enfoca en el producto final obtenido y no en el proceso que se desarrolla a lo interno del grupo durante el análisis y búsqueda de la solución al problema. Este fue un aspecto de preocupación para los docentes por el tiempo que consume esta actividad, de esta manera es importante considerar algunas alternativas que puedan apoyar $\mathrm{y}$ acompañar en este proceso a los docentes, entre ellas se pueden considerar: disminuir la cantidad de grupos de trabajo que atiende cada profesor (esto se logra aumentando el número de estudiantes por grupo de trabajo), incorporar nuevas figuras docentes como asistentes académicos, o tutores (estudiantes de cursos más avanzados que dedican algunas horas dando consulta a los estudiantes), analizar el desglose de horas dedicadas al curso de manera presencial, etc, de tal manera que este aspecto pueda ser realizado adecuadamente.

- $\quad$ Se debe promover que los estudiantes presenten a todo el grupo en forma oral los avances y el resultado final de su trabajo, esto enriquece la capacidad de expresión oral, la participación en el grupo y el aprendizaje de todos los estudiantes, y debe verse como tiempo de clase invertido en el proceso de aprendizaje, además debe ser adecuadamente valorado.

- Al final de cada proceso en grupo, sean retos, tareas o proyectos, los estudiantes deben realizar un proceso de auto-evaluación y evaluación por pares para medir el esfuerzo y compromiso de cada uno de los miembros del grupo. Este proceso contribuirá a que cada miembro analice en forma crítica su participación y entienda la responsabilidad individual implícita en el trabajo colaborativo, de igual manera es útil como medio para regular el aporte de cada estudiante al logro de la tarea. Estos procesos de evaluación deben estar basados en rúbricas claras que orienten a los estudiantes en los aspectos fundamentales a considerar. Además para facilitar su aplicación y seguimiento por parte del docente se sugiere el uso de alguna herramienta tecnológica como Google forms o un pequeño sistema informático. Ambas opciones han sido probadas durante el desarrollo del proyecto con buenos resultados.

- $\quad$ Se sugiere que para trabajar propiamente en el proyecto sean los estudiantes los que decidan la conformación de los grupos y que estos sean de 3-5 miembros, además reforzar el uso de roles e intercambio de estos en el desarrollo del trabajo, así como orientarlos en la resolución de conflictos.

\section{CONCLUSIONES}

La propuesta presentada busca generar cambios en la forma de abordar los cursos de programación de la carrera. Es sabido que los cambios implican tiempo, tanto académicos como estudiantes deben ajustar su cultura y se requieren procesos de asimilación de las nuevas estrategias didácticas. En consecuencia, se propone el cambio como un proceso fundamentalmente basado en experiencias de aprendizaje positivas tanto para los docentes como los estudiantes. También es importante tener claro que la enseñanza basada en problemas y proyectos (POPP) presupone una estrategia educativa integral y no únicamente un complemento en el proceso de aprendizaje.

A través de esta experiencia la Escuela de Informática está incorporando paulatinamente el enfoque PBL en su programa de pregrado. Sin embargo, esta transición no es fácil. En las universidades, en general, el enfoque tradicional centrado en el docente prevalece $y$ es difícil de cambiar, debido 
principalmente a las concepciones de los estudiantes sobre los procesos de enseñanza y aprendizaje, a su falta de preparación para avanzar hacia modelos centrados en los estudiantes, y a la frecuente carencia de formación pedagógica de los docentes que les dificultan adoptar nuevas estrategias didácticas. Esto conlleva a que algunos de ellos sean reacios a adoptar el enfoque y los que lo aceptan pueden no tener el conocimiento adecuado para hacer que funcione. En general, tanto los estudiantes como los docentes pueden mostrar resistencia, pueden sentirse confundidos y frustrados. Los estudiantes tienen que desarrollar nuevas estrategias de aprendizaje y los docentes necesitan desarrollar nuevas concepciones de la enseñanza. Así el reto es que los académicos aprendan cómo diseñar un conjunto de actividades de aprendizaje, donde se promuevan las distintas habilidades que el enfoque PBL puede facilitar.

Además, aunque la mayoría de los docentes manifiestan estar abiertos a hacer cambios que mejoren el aprendizaje y motiven más a los estudiantes, es evidente que estos procesos implican más trabajo y un cambio de cultura de los actores involucrados. Además es claro que se requiere de una línea de gestión clara y de una estructura de gestión curricular flexible que permita modificar las estructuras de evaluación tradicionales. La experiencia confirma que una de las mejores contribuciones que la Escuela de Informática puede ofrecer al mercado costarricense TIC son estudiantes graduados con altos niveles de autonomía. En esta línea es nuestra percepción que los planes de estudios de la Escuela deben modificarse para incluir experiencias de aprendizaje que desafien a los estudiantes a desarrollar habilidades de aprendizaje auto dirigido, así como habilidades de resolución de problemas y trabajo colaborativo. Estas experiencias deben ser cuidadosamente diseñadas e implementadas para minimizar las reacciones negativas entre los estudiantes y docentes.

En general, los resultados indican la importancia de avanzar los procesos de enseñanza y aprendizaje hacia un enfoque de PBL, ya que puede ofrecer a los estudiantes mayores posibilidades de tomar decisiones cuando se trabaja con la solución de problemas, y la forma de abordar el problema, y como consecuencia aumentar su motivación en el proceso de aprendizaje y de asumir la responsabilidad de este proceso. Los resultados también muestran que el proceso de desarrollo e implementación de metodologías de enseñanza como PBL es muy exigente, requiere mucho tiempo y esfuerzo, a veces frustrante.

A pesar de que la propuesta presentada en este artículo es muy concreta y específica para cursos del área de programación, creemos que esta no es excluyente de ser analizada por otros programas y áreas de conocimiento, y los aspectos generales de la misma pueden ser considerados en otras áreas disciplinarias.
Existen aspectos clave que son importantes para el éxito y la contribución de los principios del POPP a un plan de estudios, y que de alguna manera fueron el foco de las intervenciones realizadas y que se obtienen como resultado de las mismas, los cuales son listados a continuación:

- Es fundamental capacitar a los académicos en los principios del POPP que quieren ser implementados en los cursos.

- Los problemas/proyectos a resolver en los cursos deben estar estrechamente relacionados con las futuras actividades profesionales de los estudiantes. Deben ser ejemplares y abiertos con el fin de incentivar a los estudiantes a formular, investigar $\mathrm{y}$ resolver adecuadamente.

- Para que el trabajo en grupo sea eficaz, los estudiantes deben estar preparados para tomar y compartir responsabilidades y hacer frente adecuadamente a los conflictos.

- La responsabilidad compartida entre docentes y estudiantes en el proceso de aprendizaje se debe fomentar desde los primeros cursos de la carrera. Los estudiantes pueden ir adquiriendo diferentes niveles de autonomía adecuados a sus capacidades y conforme vayan avanzando en la carrera.

- En el desarrollo de los proyectos es necesario contar con la supervisión frecuente y oportuna de los profesores.

- El número de integrantes de los grupos de estudiantes debe ser mayor a dos personas para promover de esta forma las habilidades de los estudiantes en el trabajo en grupo y para equilibrar el esfuerzo de los docentes en dar supervisión y realimentación a los grupos.

- Debe fomentarse la responsabilidad y el compromiso de los estudiantes en el proceso de aprendizaje. Una forma de contribuir a esto son los esquemas de autoevaluación y evaluación por pares.

- Debe promoverse el trabajo colaborativo entre los docentes.

Con respecto a esto último, se deben proporcionar las condiciones adecuadas para que se fortalezca el trabajo en grupo entre los docentes, ya que es fácil deducir que un factor clave de la intervención propuesta reside en la capacidad de elaborar o seleccionar problemas que sirvan como punto de arranque para un proceso de aprendizaje que promueva todas las habilidades requeridas en los estudiantes. Idealmente este proceso debe ser trabajo en equipo, que permita darle unidad a la secuencia de los cursos y asegure un crecimiento incremental en los objetivos de aprendizaje. Se propone que, como grupo, los docentes puedan elaborar un conjunto de proyectos para cada uno de los cursos de tal manera que se consolide una base de datos de proyectos que puedan ser implementados y de los cuales los estudiantes puedan elegir. Así se aseguraría, por un lado, que los proyectos respondan a los contenidos y objetivos de los cursos y por otro, que los estudiantes se motiven al escoger el problema que les resulte más interesante. 


\section{RIESGOS}

Cualquier proyecto de cambio siempre se enfrenta a obstáculos. Durante el proceso de investigación-acción se pusieron en evidencia algunas de las problemáticas a las que se enfrenta una iniciativa como la propuesta: (1) inestabilidad del cuerpo docente: los docentes varían de un semestre al otro, y los cursos muchas veces están en manos de docentes con jornadas parciales, lo que no garantiza la dedicación necesaria para el logro de los objetivos propuestos; (2) falta de liderazgo positivo en el área de programación que logre darle unidad y coherencia a los cursos tanto desde el punto de vista técnico como pedagógico; (3) escasa comunicación positiva entre los docentes y dificultades para consolidar un trabajo grupal efectivo; (4) falta de una directriz clara de la administración de la unidad académica con respecto a la incorporación de este tipo de enfoques y a la "obligatoriedad" de los docentes a seguirla.

Es claro que estas condiciones deben ser atendidas y deseablemente subsanadas para poder garantizar un proceso de cambio efectivo y permanente que contribuya a formar graduados que respondan de una mejor manera a las demandas de la sociedad actual.

\section{AGRADECIMIENTOS}

Agradecemos la participación en este proceso a la profesora Marianne Lykke, de la Universidad de Aalborg, Dinamarca quien participó en el diseño de la propuesta de intervención, y durante el proceso mismo. De igual manera a los docentes de la Escuela de Informática, particularmente de las cátedras de Ingeniería de Sistemas, Bases de Datos y Programación que participaron en los procesos de formación docente y que con su experiencia contribuyeron a lograr una mejor comprensión del contexto en el que se diseña esta propuesta, y a todos los estudiantes de los cursos EIF 200 Fundamentos de informática, EIF 201 Programación I, EIF 204 Programación II, y EIF 206 Programación III, que participaron del proceso durante los ciclos lectivos en que se llevó a cabo la intervención educativa.

\section{REFERENCIAS}

[1] F. Dochy, M. Segres, Van den Bossche, P., and D. Gijbels, "Effects of Problem-Based Learning: A Meta-Analysis," Learn. Instr., vol. 3, pp. 533-568, 2003.

[2] L. Prieto, "Aprendizaje activo en el aula universitaria: el caso del aprendizaje basado en problemas," Miscelánea Comillas Rev. Cienc. Humanas Soc., vol. 64, no. 124, pp. 173-196, 2006.

[3] J. Mills and D. Treagust, "Engineering Education - Is Problem-Based or Project-Based Learning the Answer?," Australas. J. Eng. Educ., vol. 4, 2003.

[4] A. Kolmos, "Estrategias para desarrollar currículos basados en la formulación de problemas y organizados en base a proyectos," Educar, vol. 33, pp. 77-96, 2004.
[5] Universidad Nacional, "Modelo Pedagógico," 2007.

[6] S. Barge, "Principles of problem and project basic learning. The Aalborg PBL Model," Aalborg University., Aalborg, 2010.

[7] M. Prince and R. Felder, "Inductive teaching and learning methods: Definitions, comparisons, and research bases," $J$. Eng. Educ., vol. 95, pp. 123-128, 2006.

[8] M. Prince and R. Felder, "The Many Faces of Inductive Teaching and Learning," J. Coll. Sci. Teach., vol. 36, no. 5, 2007.

[9] J. Perrenet, P. Bouhuijs, and J. Smits, "The suitability of problem-based learning for engineering education: theory and practice," Teach. High. Educ., vol. 5, no. 3, pp. 345-358, 2000.

[10] A. Kolmos, "Estrategias para desarrollar currículos basados en la formulación de problemas y organizados en base a proyectos," Educar, vol. 33, pp. 77-96, 2004.

[11] E. Nuutila, S. Törmä, and L. Malmi, "Pbl and computer programming - the seven steps method with adaptations," Comput. Sci. Educ., vol. 15, no. 2, pp. 123-142, 2005.

[12] P. Kinnunen and L. Malmi, "Problems in Problem-Based Learning - Experiences, Analysis and Lessons Learned on an Introductory Programming Course," Inform. Educ., vol. 4, no. 2, pp. 193-214, 2005.

[13] Hamalainen, "Problem-based learning of theoretical computer science \}," 2004, vol. 3, pp. 20-23.

[14] T. Guskey, "Professional Development and Teacher Change," Teach. Teach. Theory Pract., vol. 8, pp. 381-391, 2002.

[15] Stewart, R.A., "Investigating the link between self-directed learning readiness and project-based learning outcome: The case of international masters students in an engineering management course.," European Journal of Engineering Education, vol. 4, no. 32, pp. 453-465.

[16] ACM, "Computer Science Curricula 2013," ACM, 2011.

[17] OIT, "Una fuerza de trabajo provista de formación para un crecimiento sólido, sostenible y equilibrado," Ginebra, 2010.

[18] World Bank, "Lifelong Learning in the Global Knowledge Economy: Challenges for Developing Countries," The World Bank, Washington, D.C., 2003.

[19] World Bank, "Constructing Knowledge Societies: New Challenges for Tertiary Education," 2002. [Online]. Available: www.worldbank.org/education.

[20] Bourne,T., "Assessing reflective learning," Education Training, 45(5), vol. 5, no. 45, pp. 267-271.

[21] J. S. Brown and P. Duguid, The social life of information. Boston: Harvard Business School Press, 2000.

[22] L. Dynan, T. Cate, and K. Rhee, "The impact of learning structure on students' readiness for self-directed learning," $J$. Educ. Bus., vol. 84, no. 2, pp. 96-100, 2008.

[23] Weimer, M., Learner-centered teaching: five key changes to practice. San Francisco,CA: Jossey- Bass., 2002.

[24] Raidal,S.L and Volet,S., "Preclinical students' predispositions towards social forms of instruction and self-directed learning: a challenge for the development of autonomous and collaborative learners.," Higher Education, vol. 57, pp. 577596, 2009.

[25] A. Finch, "Autonomy: Where are we? Where are we going?," presented at the JALT CUE-SIG, 2002 Proceedings, 2002, vol. 15 .

[26] W. Y. Hsu, "Online education on campus: A technological frames perspective on the process of technology appropriation," Unpublished Doctoral Thesis, University of London, London, 2002. 
[27] M. E. Calzadilla, "Aprendizaje colaborativo y tecnologías de la información y la comunicación," Rev. Iberoam. Educ., vol. 1, no. 10, 2002.

[28] R. T. Johnson and D. W. Johnson, "Action research: Cooperative learning in the science classroom," Sci. Child., vol. 24, pp. 31-32, 1986.

[29] N. Scagnoli and M. Stephens, "Collaborative learning strategies in online education," presented at the Online Conference for Teaching and Learning (IOC2005), Illinois, 2005.

[30] D. Rios, "Sentido, criterios y utilidades de la evaluación del aprendizaje basado en problemas," Educ. Médica Super., vol. 21, no. 3, 2007.

[31] J. Biggs, Calidad del aprendizaje universitario. Madrid: Narcea, 2005

[32] D. Rios and P. Troncoso, "Autoevaluación de los alumnos: una estrategia participativa orientada al aprender a valorar," Rev. Estud. Exp. En Educ., vol. 2, no. 4, pp. 111-120, 2003.

[33] M. A. Zabalza, "Evaluación de los aprendizajes en la Universidad," in Didáctica Universitaria, García Valcárcel, Ed. Madrid: La Muralla, 2001.

[34] Descombe, M., The Good Research Guide: for small-scale social research projects., 4ta edición. MacGraw Hill, 2010.

[35] M. Coto, S. Mora, and M. Lykke, "Design considerations for introducing PBL in Computer Engineering," Informática CLEI 2012 XXXVIII Conf. Latinoam., pp. 1-10, 2012.

[36] M. Coto, S. Mora, and M. Lykke, "Developing the Qualifications of the ICT Workforce through Problem-Based Learning," in Changing Education through ICT in Developing Countries, M. Georgsen and P. Zander, Eds. Aalborg University Press, 2013, pp. 33-60.

[37] Coto,M., Mora,S., and Alfaro,G., "Giving more autonomy to computer engineering students: are we ready?," presented at the IEEE Global Engineering Education Conference (EDUCON), Istambul, Turkey, 2013.

[38] S. Mora and M. Coto, "Curriculum Integration by Projects: Opportunities and Constraints. A Case Study in Systems Engineering," presented at the XXXIX Latin American Computing Conference (CLEI), Venezuela, 2013.

[39] S. Butler, "Problem based learning at NCSU," 2002.

[40] Chan,V., "Readiness for Learner Autonomy: What do our learners tell us?," Teach. High. Educ., vol. 6, no. 4, pp. 505518, 2001.

[41] Vermunt, J.D. and Verloop, N., "Dissonance in students' regulation of learning processes.," European Journal of Psychology of Education, vol. 15, no. 1, pp. 75-89, 2000. 